\title{
Improving Learning Tasks for Mentally Handicapped People Using AmI Environments Based on Cyber-Physical Systems
}

\author{
Diego Martín (D), ${ }^{1}$ Borja Bordel $\left(\mathbb{D},{ }^{1}\right.$ Ramón Alcarria $\mathbb{D},{ }^{2}$ and Yone Castro ${ }^{3}$ \\ ${ }^{1}$ ETSI Telecommunications, Universidad Politécnica de Madrid, Av. Complutense 30, 28040 Madrid, Spain \\ ${ }^{2}$ ETSI Topography, Geodetics and Cartography, Universidad Politécnica de Madrid, Camino de la Arboleda s/n, \\ 28031 Madrid, Spain \\ ${ }^{3}$ Fundación PRODIS, Bulevar Indalecio Prieto 2, 28032 Madrid, Spain \\ Correspondence should be addressed to Diego Martín; diego.martin.de.andres@upm.es
}

Received 28 July 2017; Accepted 26 November 2017; Published 28 January 2018

Academic Editor: Pino Caballero-Gil

Copyright ( 2018 Diego Martín et al. This is an open access article distributed under the Creative Commons Attribution License, which permits unrestricted use, distribution, and reproduction in any medium, provided the original work is properly cited.

\begin{abstract}
A prototype to improve learning tasks for mentally handicapped people is shown in this research paper using ambient intelligence techniques and based on cyber-physical systems. The whole system is composed of a worktable, a cyber-glove (both with several RFID and NFC detection zones), and an AmI software application for modeling and workflow guidance. A case study was carried out by the authors where sixteen mentally handicapped people and 3 trainers were involved in the experiment. The experiment consisted in the execution of several memorization tasks of movements of objects using the approach presented in this paper. The results obtained were very interesting, indicating that this kind of solutions are feasible and allow the learning of complex tasks to some types of mentally handicapped people. In addition, at the end of the paper are presented some lessons learned after performing the experimentation.
\end{abstract}

\section{Introduction}

Cyber-physical systems (CPS) are intersections of the physical and the cyber worlds [1]. Although different definitions and proposals may be found [2], basically, these new systems allow creating feedback control loops where sensors and actuators obtain information about the real world, which is processed by new generation cyber components (as, e.g., enhanced pattern-recognition techniques), in order to infer the state of the environment and its inhabitants and be able to act to reach a more sustainable and profitable situation [2].

With these ideas, one of the most common applications for CPS is traceability [3]. Both product traceability and people surveillance have been enhanced through the use of CPS in the last years [4]. Moreover, some pedagogical works have proved that this kind of systems are useful to control the student learning in the so-called Industry 4.0 educational scenarios. However, these solutions require a high number of deployments, a complete remodelation of teaching methodologies, and manners. Therefore, usually they are referred as "the systems of the future," discarding their viability nowadays.

Nevertheless, some small deployments or interventions may be useful in certain scenarios, such as in educational environments involving people who are mentally handicapped. These solutions do not fit totally the requirements of the new generation systems but follow their principles employing the existing technology. In the context of ambient intelligence (AmI) systems, for example, this approach is being investigated in a very successful way [5]. In particular, we argue that it is a very promising view for scenarios where traditional techniques are insufficient, but where total automation (as CPS paradigm proposes) is not valid at all (i.e., the human actor must endure). This is the case, for example, of the applications involving people with special needs.

This paper is an extended version of our previous work [6], which proposed an enhanced ambient intelligence (AmI) system based on cyber-physical systems for tracking the tasks being performed by people who are mentally handicapped during their learning process. 
The CPS presented in this research work include a cyberglove, a worktable, and an AmI software for creating and controlling workflows, whose tasks involve actions about the cyber-glove and the worktable. This system is framed in a research project funded by a grant from Madrid Autonomous Community called SEMOLA. Currently, this research project is in a preliminary phase; so the developments done are also at an exploratory stage. The whole system is called LAoCA, an acronym from "Learning Architecture over CPS and AmI."

The objective of this research work is to validate whether an AmI environment for CPS traceability facilitates learning for mentally handicapped people and improves control over tasks and the acceptance of the system by users. Is not the objective of this paper to validate the characteristics of the system with which the experimentation was carried out, which could be a possible future work.

In order to guide this research work, three research questions were stated:

(i) RQ1: Can an AmI environment for CPS traceability facilitate learning for mentally handicapped people?

(ii) RQ2: Does it improve control over the performed tasks?

(iii) RQ3: Is LAoCA accepted by the final users?

A case study was carried out by the authors in order to validate the environment and the solution proposed in this paper, where sixteen mentally handicapped people with Down syndrome from one of the most important foundations for mentally handicapped people in Spain were involved. The validation consisted of studying the improvement of participants' learning capabilities, analyzing the system's control of the tasks performed using the cyberglove and the worktable, and performing a survey about the final user acceptance of the system.

The results obtained are very promising confirming that the proposed solution is feasible, but as it is a preliminary version. This first validation allowed us to find weaknesses in the proposal with the aim of improving it, in following versions and improving the design validation for future experiments.

\section{Related Work}

As said before, cyber-physical systems paradigm fits perfectly the requirements of traceability solutions [3]. In fact, various previous works have investigated the use of CPS (and other similar solutions such as Smart Environments [7]) in AmI solutions and traceability systems.

In particular, the National Institute of Standards and Technology (NIST) has proposed a reference architecture valid to be employed as framework for traceability solutions and industrial automatic systems [8]. This proposal aims to turn into the basic document for the future commercial CPS. For example, following this line, some research works have investigated the use of low-energy devices in order to display information in different scenarios such as supermarkets, production systems, or hospitals $[9,10]$. The basic idea is to employ new, open, and more efficient technologies such as the electronic ink in order to improve the current automatic industrial systems based on proprietary technologies [11, 12], programmable logic controller (PLC) $[13,14]$, and very lowlevel protocols such as OPC (Object Linking and Embedding for Process Control) [15].

On the contrary, CPS approach refers an integrated solution including all the requirements needed for automated industrial processes, allowing a reduction in the system's complexity, and therefore, reducing the investment. Using this framework, some traceability systems have been proposed [3], proving that the number of errors committed by workers gets reduced in comparison to traditional systems. Moreover, solutions focused on inferring the activities being performed by people in certain scenarios (as, e.g., the daily living) have been reported [16]. In these proposals (usually), the enabling technology is radio frequency identification (RFID), which is employed to create cyber-gloves, bracelets, and other instruments [17, 18]. These designs, nevertheless, are usually very obtrusive, so the execution of tasks and processes is highly modified when considering the use of these tools. For this reason, learning solutions have not included this kind of systems yet (as they may impede the development of some activities). This idea, which is valid in general, is much more important and critical when people who are handicapped are considered. Despite the effort of the NIST (and other standardization organizations) to define the concept of CPS, several different proposals and architectures may be found in the literature [2]. Nowadays, even the appearance of the Industry 4.0 systems [19] in Germany (based on CPS solutions and principles) has turned the scene more complex and heterogeneous.

In fact, the Industry 4.0 revolution aims to modify the entire society. This revolution includes learning and teaching. In this context, different proposals about how the future learning systems (based on Industry 4.0 and CPS paradigms) could be constructed and implemented may be found. Systems based on gamification and virtual reality are the most common. However, other proposals based on defining adaptive systems being able to facilitate the learning of, for example, new tasks in industrial scenarios have been reported [20].

Finally, in the last five years, the CPS paradigms have been also applied to learning systems. In particular, as we said, several works try to infer the Activities of Daily Living [21] performed by users using a sort of RFID-enabled cyber-glove [22] (possibly complemented with additional sensors such as accelerometers [23]), connecting the outputs of these tools to a system which helps people to learn in a faster way (by means of real-time feedback, assisted living, etc.). The applications of these systems are mainly focused on occupational therapy [24] for neurological patients; children control, augmented reality [25], and behavioral therapy [26]; and accessibility (such as the system SignAloud proposed by the MIT [27]).

\section{LAoCA (Learning Architecture over CPS and AmI)}

In this section, we present a second version of our previous work [6], a development framed within a research project 
called SEMOLA (2016-2019), funded by a grant from Madrid Autonomous Community. Among the many specific objectives of the SEMOLA project, we developed a prototype to test the technology to support people with intellectual disabilities in their personal development, taking timely remedial actions and anticipating problems that can be found. Thus, we will generate enough knowledge for the upcoming project phases, such as lessons learned and best practices.

Our LAoCA (Learning Architecture over CPS and AmI) prototype is composed of three elements: a cyber-glove, a worktable for tracing the items, and an AmI software application for creating, managing workflows, and also controlling the actions performed with the whole system. All components are connected by means of a Bluetooth network with star topology, acting the AmI application as central element. Bluetooth 3.0 technology allows creating ad hoc networks with a valuable data rate (until $3 \mathrm{Mbps}$ ), which perfectly fit with the real-time requirements of $L A o C A$. In the next section, we are reviewing in detail each cited component.

3.1. Worktable. In this section, we present a worktable made of plastic with four detection zones as shown in Figures 1 and 2; Figure 3 shows the tagged elements to be detected by the worktable. The main purpose for which the worktable was designed is to identify objects (tagged with RFID or NFC) placed in the four detection zones and transmit that information wirelessly in real time to the control program. It also may detect the moment when an object previously placed over the worktable is removed. The worktable also includes some actuators in order to interact with the users and inform them about the execution of the planned tasks (Section 3.3).

The electronics of the worktable are composed by the following: four printed coils working as antennas which sense the four detection areas; four RDM8800 RFID and NFC readers (one for each detection area); five Arduinos Nano, four dedicated to manage the four antennas and the last one acting as main microcontroller in the system; a bluetooth module for its communication with the control software; four green LEDs (one for each detection zone) used to illuminate the detection surface, and additional three LED indicators (actuators) for showing its operation and giving some feedback to the user. The surface LEDs are used to describe the task to be performed at an earlier stage of training. Figure 4 shows an inside view of the worktable, while Figure 5 shows the electronic schema of the worktable.

3.2. Cyber-Glove. Figure 6 shows the prototype of the cyberglove proposed in this research paper. It is made of cloth, and it has a compartment where all the necessary electronics for its operation are stored (Figure 7). This compartment can be positioned below or above the wrist in order to facilitate hand movements.

The main functionality of the cyber-glove is to be a device capable of identifying objects tagged with RFID or NFC wirelessly and having communication capabilities with the

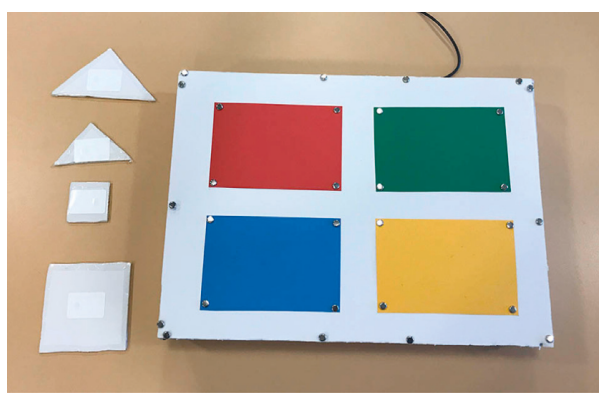

Figure 1: Worktable with four detection zones and NFC tags.

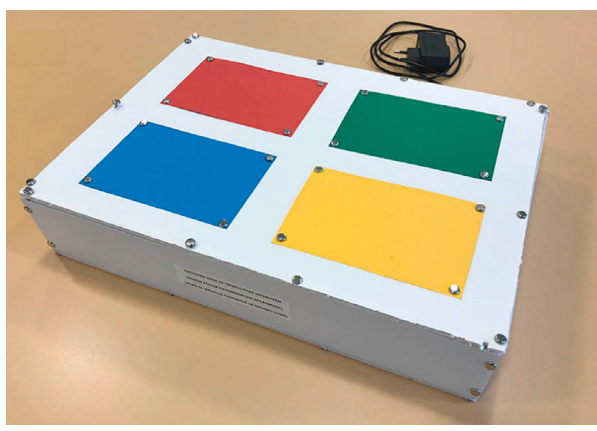

FIgURe 2: Perspective view of worktable.

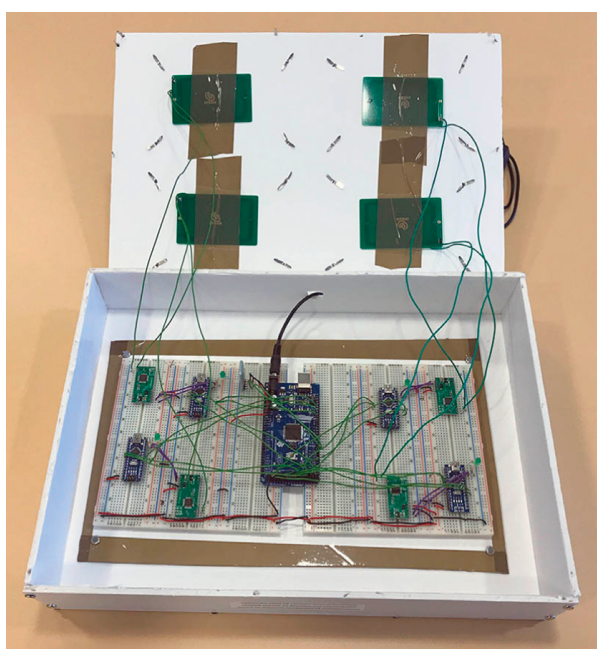

Figure 3: Tagged elements.

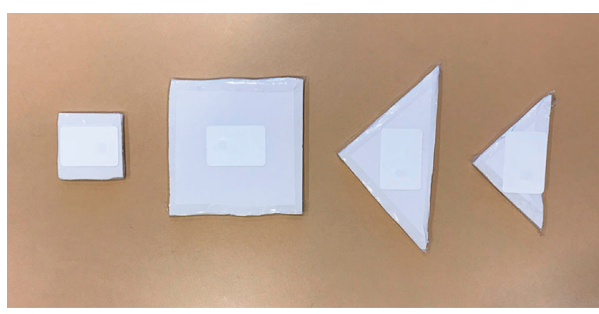

FIgURE 4: Electronics inside the worktable.

software that controls and manages the movements. In summary, the cyber-glove may detect any object touched or taken by users and send the information immediately to the 


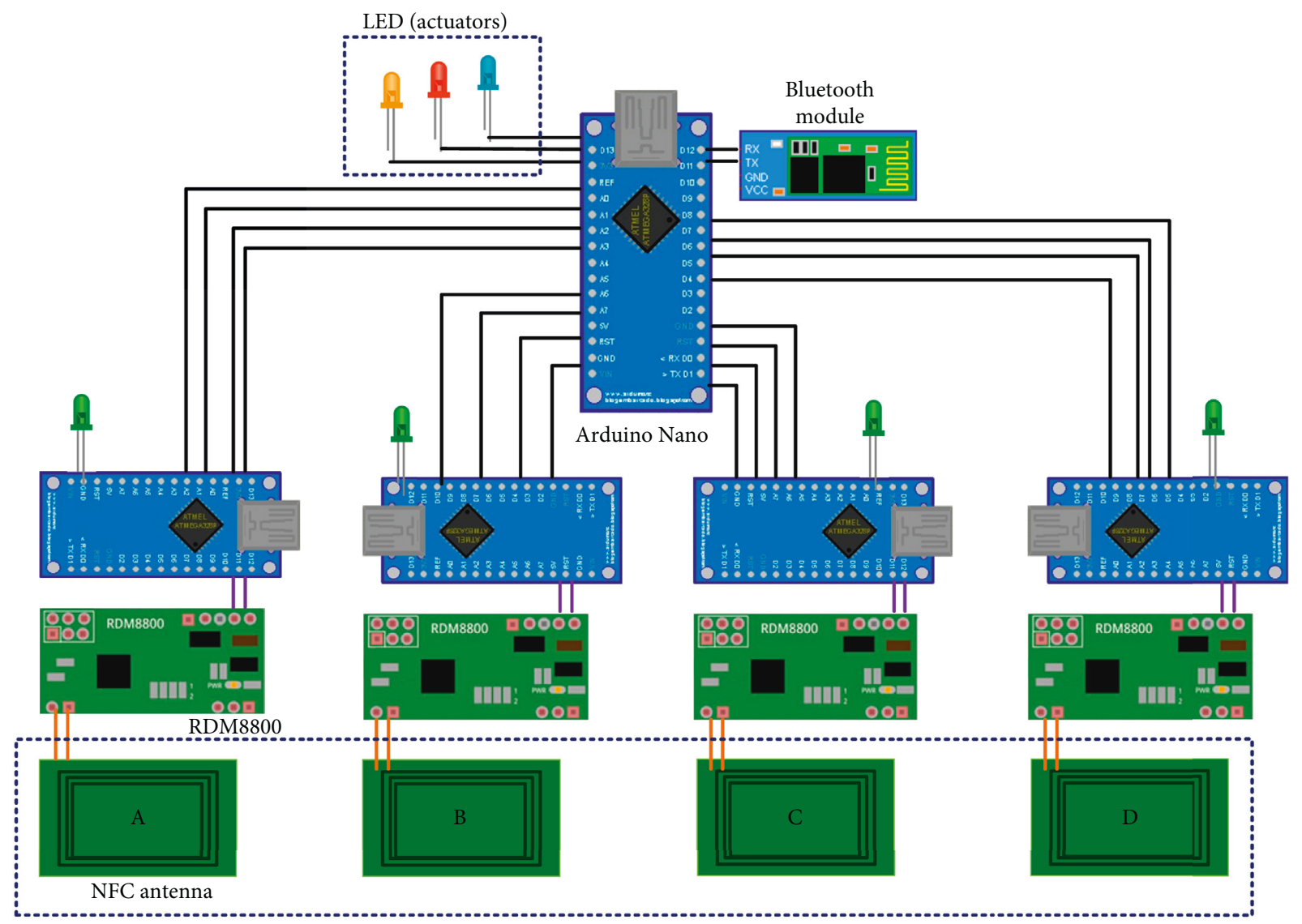

RFID sensors

FIGURE 5: Worktable electronics schema.

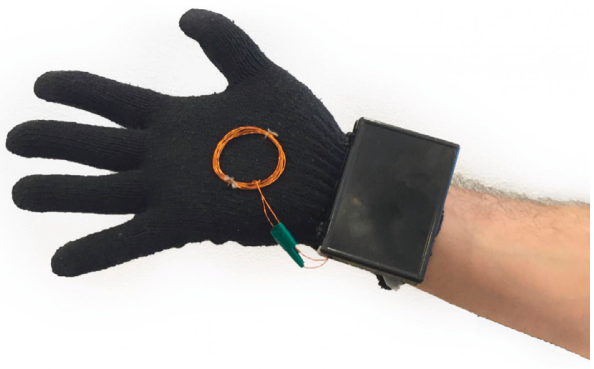

Figure 6: Cyber-glove.

management software. The cyber-glove may also detect the moment when the user releases the object (if he held it) informing to the management software. The cyber-glove cannot detect two or more objects touched at the same time.

The electronics of the cyber-glove consists of several elements as seen in Figure 7: an Arduino Nano for data processing, a RDM8800 NFC chip for reading the RFID and NFC elements, an HC-06 Bluetooth module for communicating with the control program, a coil in the palm that works as antenna for reading RFID and NFC elements, and a battery.

3.3. Workflow Creation and Control Software. The workflow creation and control software is an ambient intelligent tool for creating and managing workflows made of different tasks (such as position control or state monitor manage) involving the tagged objects with RFID and NFC through the cyberglove and the worktable. The different tasks are related by means of some "transitions" which are triggered, if the associated condition is fulfilled.

This software has two main features: (a) it models the workflow of the task intended to execute, using a prosumer interface that allows a user with no programming experience to model the tasks, and (b) it also executes the control workflows previously created in order to supervise and analyze the actions performed by a user who is working with the cyber-glove and the worktable.

For workflow creation, the AmI tool includes a graphic environment where users may compose their own workflows using different predesigned modules. In respect to the second functionality (workflow control), the software permanently is hearing for the information from the cyber-glove and/or the worktable. It evaluates the notifications and triggers the proper transitions (if any exists). Every workflow should include "fatal error tasks" to which the workflow moves if the execution fails. Besides, a "successfully finished task" would be advisable to be included. In Figure 8, we present an example workflow, indicating the described desirable structure.

Additionally, the AmI software transmits towards the worktable information about the workflow evolution. Then, 


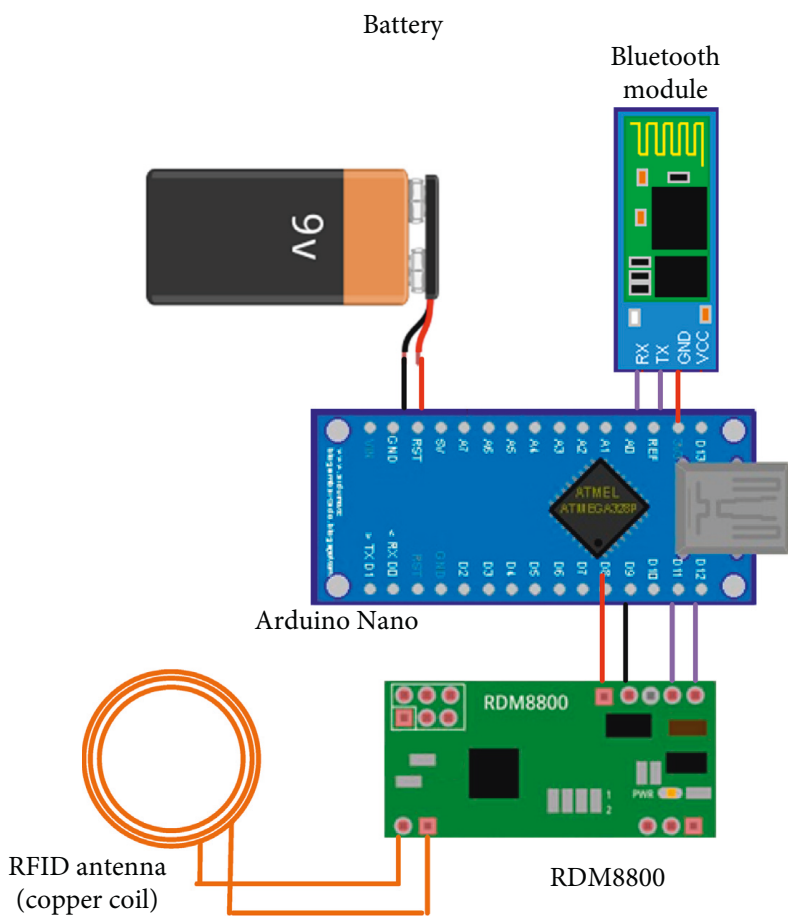

FIgURE 7: Cyber-glove electronics schema.

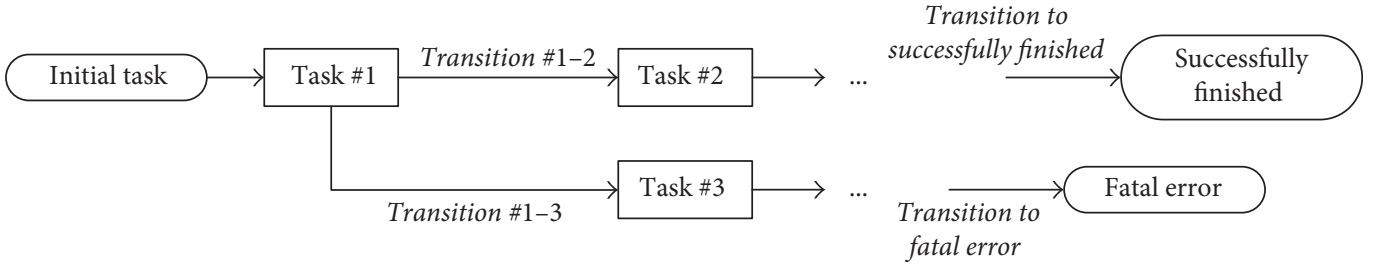

FIgURE 8: Example workflow.

the worktable can show that information (using the actuators-LEDs) to the users (e.g., if the workflow finishes with a fatal error, a red LED will be turned on).

\section{Experimental Validation}

This research work has been guided by three research questions presented in the introduction. We designed and performed a case study where sixteen mentally handicapped people performed some tasks, defined and supervised by three trainers from a foundation committed to people with intellectual disabilities. The validation presented in this research work is a preliminary action framed in the early years of development of the SEMOLA project. Our goal is to test the feasibility of LAoCA and, with the results obtained, improve it with a new deployment at the foundation and extend the experimentation with more people involved. The researchers did not influence participants using the environment. This approach is appropriate to replicate the experiment in similar contexts.

4.1. Context. The authors of this research work (hereafter, experts) designed, executed, and assessed the case study, whose research areas are cyber-physical systems, ambient intelligence, knowledge management, etc.

Sixteen mentally handicapped people from the foundation (hereafter, participants) participated in the experimentation performing 4 tasks each, and three trainers from the same foundation helped in training the participants in some tasks using the software for workflow modeling of LAoCA.

Sixteen participants conformed the sample. The sample meets the criteria of accessibility, suitability, and representativeness. All of them are potential users of the system proposed in this research work. The 16 participants were grouped into four groups depending on their type of disability, and the characteristics of the participants are described in Table 1. Participants were selected by the experts to perform the experimentation following the characteristics described in Table 1.

It is considered that the sample is representative since the average age is located in the general middle point of development of people with intellectual disabilities, taking into account that $80 \%$ of the sample has Down syndrome. None of the participants developed cognitive impairment (evaluation through CAMCOG [28]). The degree of disability and 
TABLE 1: Summary of the main features of the participants (own elaboration).

\begin{tabular}{|c|c|c|c|c|c|c|c|}
\hline Level & $\begin{array}{c}\text { Age } \\
(\text { mean })\end{array}$ & $\begin{array}{l}\text { Type of } \\
\text { disability }\end{array}$ & $\begin{array}{c}\text { Disability } \\
(\%) \\
(33-100) \\
\end{array}$ & $\begin{array}{l}\text { Limitation on the } \\
\text { global activity }(\%) \\
(0-100)\end{array}$ & $\begin{array}{l}\text { Cognitive impairment } \\
\text { (low-moderate-severe) }\end{array}$ & $\begin{array}{c}\text { Oral comprehension } \\
\text { level (low-medium- } \\
\text { high) }\end{array}$ & $\begin{array}{c}\text { Mobility } \\
\text { (difficulty-no } \\
\text { difficulty) } \\
\end{array}$ \\
\hline 1 & 24.25 & Psychic & 64 & 61.75 & Moderate-low & Medium high & Difficulty \\
\hline 2 & 26 & Psychic & 69.75 & 64.75 & Moderate-severe & High & No difficulty \\
\hline 3 & 22.75 & $\begin{array}{l}\text { Psychic } \\
\text { sensory }\end{array}$ & 74.25 & 74.5 & Severe-moderate & Medium-low & Some difficulty \\
\hline 4 & 24.5 & $\begin{array}{l}\text { Psychic, } \\
\text { physical and } \\
\text { sensory }\end{array}$ & 87.25 & 83.25 & Severe & Low & Difficulty \\
\hline Mean & 24.38 & - & 72.75 & 71.06 & - & - & - \\
\hline
\end{tabular}

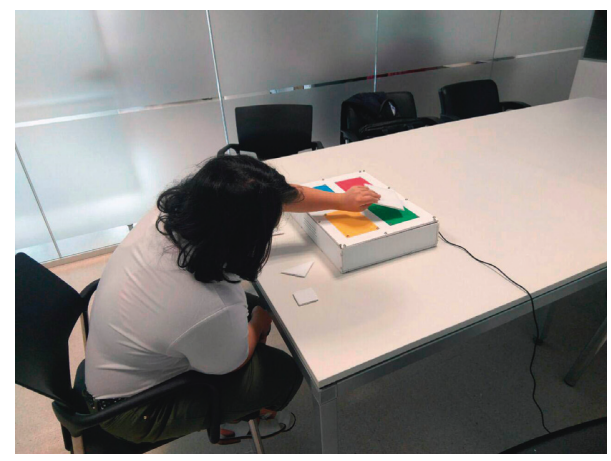

(a)

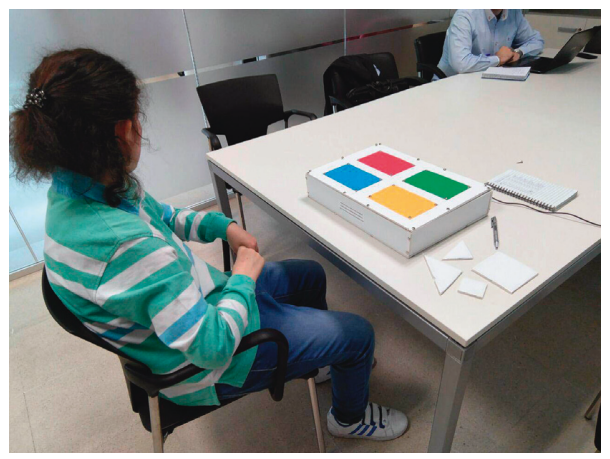

(b)

FIgURE 9: Two participants performing the experiment.

limitation in the global activity is representative of different stages of dependence, and they reflect what type of difficulties can occur in the general population with intellectual disability susceptible to use this type of technology. Representatives of different levels of oral comprehension have been employed, in order to counterbalance the results in relation to the instructions that have been used in the method. Likewise, people with different types of problems have been chosen (visual and motor) that could affect the test. Figure 9 shows two photographs of two people participating in the experiment.

The test population was selected to be as much heterogeneous as possible in order to validate (in an implicit way) the adaptation capability of the system for different users. In fact, no significant differences were observed between the system performance when employed by different users.

4.2. Planning. The experimentation plan was executed in four phases.

4.2.1. Training Phase. Trainers received some instructions about the use and operation of the CPS presented in the paper with the aim to train the participants about the use of the cyber-glove and to perform some tasks with it. The training sessions consisted of a one-hour lecture at the beginning of the experimental validation given by one of the experts. 
TABLe 2: Statistical summary of the errors committed in the tasks' execution.

\begin{tabular}{lcccccccc}
\hline \multirow{2}{*}{ Task difficulty level } & \multicolumn{4}{c}{ With LAoCA } & \multicolumn{4}{c}{ Without LAoCA } \\
& Median & SD & Min & Max & Median & SD & Min & Max \\
\hline Easy & 1 & 1.26 & 0 & 4 & 3 & 1.61 & 1 & 6 \\
Medium & 4 & 1.01 & 2 & 5 & 4 & 1.26 & 2 & 7 \\
Difficult & 5 & 1.11 & 3 & 7 & 5 & 1.5 & 3 & 8 \\
Total & 4 & 1.76 & 0 & 7 & 4 & 1.75 & 1 & 8 \\
\hline
\end{tabular}

4.2.2. Task Performing. The participants executed six different tasks: three of them were done using LAoCA and the other three without it. At the beginning of each task, the system showed the sequence of movements through a tablet where the participants observed in a visual diagram the movements to be performed with the tagged objects (shown in Figure 3) on the worktable in order to learn the task. For the experiment without LAoCA, the coach was in charge to explain the task to the participants. A task is a sequence of movements over the worktable of several tagged objects. Following the recommendations of the trainers, for this experimentation, we defined three types of tasks depending on its difficulty:

(1) Easy tasks: composed of five movements of only one tagged object

(2) Medium tasks: composed of five movements of each two tagged objects

(3) Difficult tasks: composed of eight movements of each four tagged objects.

All the tasks were randomly generated. The initial idea for experimentation was to use the three elements proposed in this research paper as described in Section 3. But, we had serious problems for the participants to use the glove correctly since most of them paid more attention to the glove than to the accomplishment of the tasks. Some participants completed the tasks with the glove and others did not, which is why we decided to remove the data obtained through the cyber-glove in the experimentation. We understand that the cyber-glove is a very intrusive element for people with mental disabilities; we will discuss this in Section 6 and conclusions.

4.2.3. Data Evaluation Phase. In this phase, the trainers were asked about the participants' impressions about the use of the system by the experts. They also helped to assess 48 tasks performed by the participants.

4.3. Data Collection. The data gathered to answer RQ1 were obtained from the software that guided the tasks and the notes taken by the experts during the "Tasks performing phase." We measured the number of errors made by the participants. For example, a wrong movement or moving the wrong object is considered an error. The data collected to address $R Q 2$ were obtained from the control software and workflow guidance of LAoCA and the perception from the trainers; at first, a survey was considered to ask the trainers, but due to small number we opted for oral interviews. The information to answer $R Q 3$ was obtained through surveys filled by the trainers after evaluating each participant.

An experiment was carried out to answer the research questions proposed in Section 1, where different mentally handicapped users, characterized by Table 1, performed different tasks described in Section 4.2.

\section{Results}

In this section, we present the results obtained from the case study. The data and information will be presented trying to answer the three research questions.

5.1. Can an AmI Environment for CPS Traceability Facilitate Learning for Mentally Handicapped People? This section presents the results from the validation phase where the participants performed 4 tasks: two of them using the proposal presented in this research work and the other two without using our proposal, that is, learning in a traditional way the actions needed to complete the workflow.

Table 2 shows a summary of the data obtained, presenting statistical values on the errors committed in the execution of the tasks. The table presents the median, the standard deviation, the minimum and maximum number of errors for each type of tasks. The median of errors using LAoCA is 4 with a standard deviation of 1.76 and a number of errors ranging from 0 to 7 , while without the use of our proposal, the median error is also 4, with a standard deviation of 1.75 and values ranging from 1 to 8 .

Figure 10 shows two boxplots comparing the distributions of errors for the tasks guided with LAoCA and without it. As we can see, they have the same value for the medians, and the standard deviation is practically the same for both cases. Nonetheless, we performed a Mann-Whitney $U$ test in order to check whether the participants perform less errors by using LAoCA. The result was negative $(p>0.05)$, indicating that there is no evidence to confirm that with our proposal, fewer mistakes are made with the execution of the tasks, as expected from Figure 10.

Continuing the analysis of the data, we realized that people with a higher level of disability made more errors in general, both using our proposal and not using it. Therefore, we decided to exclude persons with the highest level of disability from the study; that is, we removed from the study the people on level 4 as shown in Table 1. The results are shown in Figure 11. With these new data, the distributions are somewhat different as well as the values for the median. 


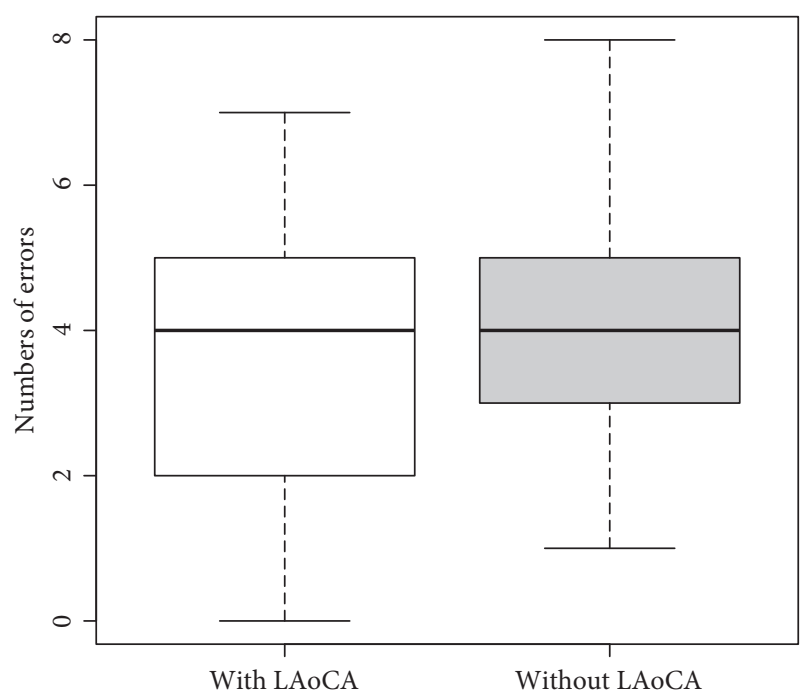

Figure 10: Error distributions for all tasks.

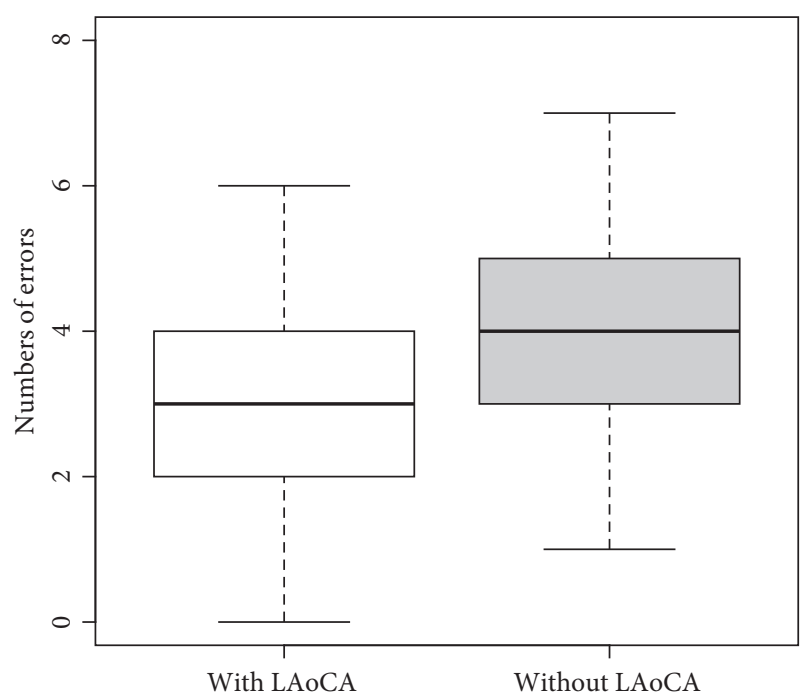

FIGURE 11: Error distributions for all tasks removing people on level 4.

The median for the group that has used LAoCA is 3 while for the group that has not used LAoCA is 4 . We performed a Mann-Whitney $U$ test to confirm if there is now a difference between the distributions, but the results were still negative $(p>0.05)$.

We conducted a more in-depth study, but this time, we decided to exclude people on levels 3 and 4 from the analysis since they were the most vulnerable to making mistakes and keeping the data obtained from people on levels 1 and 2, as shown in Table 1; the data distributions are shown in two boxplots in Figure 12. The medians remain the same as the data distributions shown in Figure 11, 3 for the group that used LAoCA and 4 for those who did not. We performed a Mann-Whitney $U$ test to confirm if there is now a difference between the distributions, and in this case, the result was positive ( $p=0.01)$. So, we can state that errors increase when not using LAoCA with people on levels 1 and 2 and all kinds of tasks: easy, medium, and difficult.

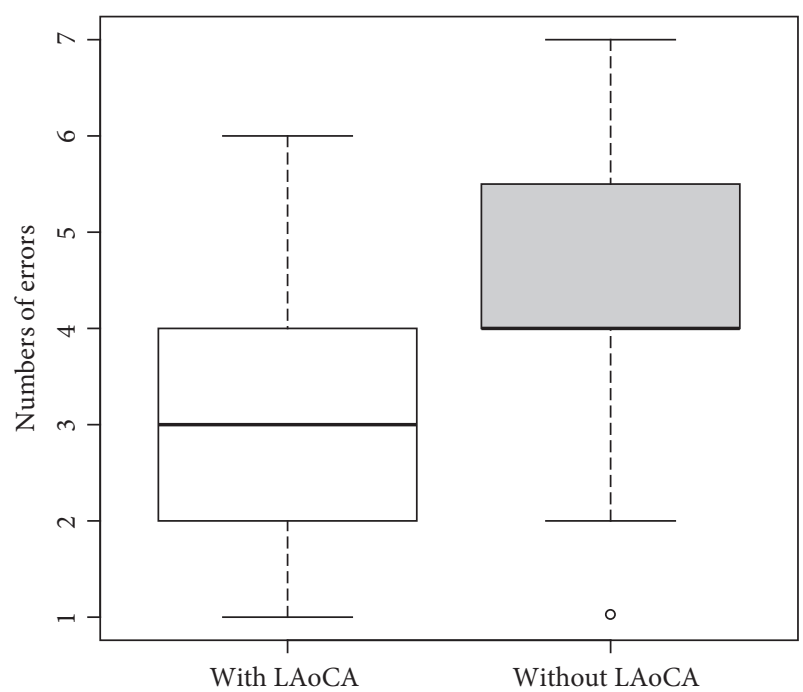

FIGURE 12: Error distributions for all tasks for the people on levels 1 and 2 .

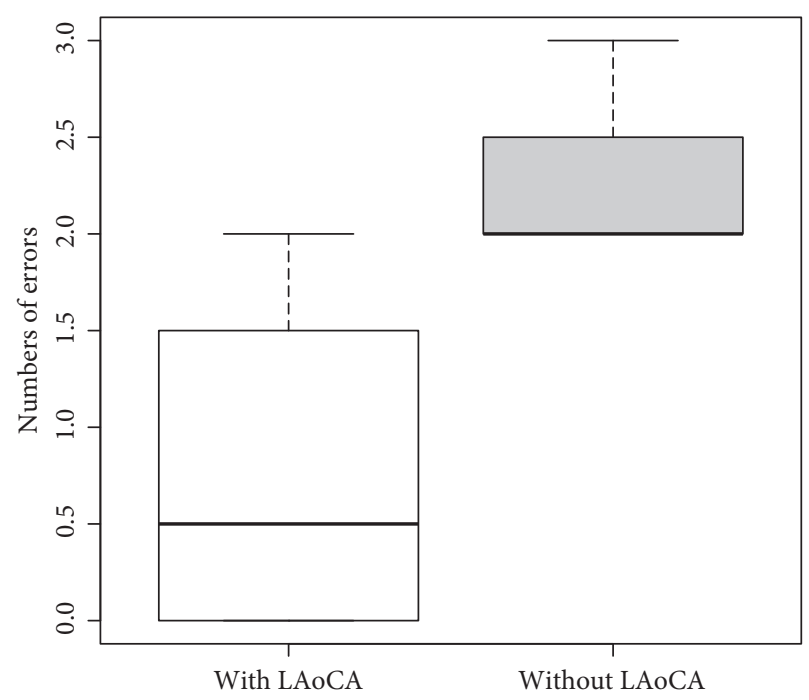

FIGURE 13: Error distributions for easy task and people on level 3.

However, we performed a further study analyzing the results for each type of task and disability levels. During the execution of the experiment and analyzing the data obtained, we realized that the participants on level 3 also improved their numbers of errors performing the task tagged as easy when using LAoCA; both distributions are shown in Figure 13. In this case, the medians are very different, 0.5 for the case of using LAoCA and 2 for the case of not using it. By performing a Mann-Whitney $U$ test, we confirm the hypothesis that the number of errors was reduced in the case of using LAoCA with the easy task and the people on level $3(p<0.05)$. In spite of this, the statistical test performed has very little power because there are very few samples that fulfill the condition of performing the easy task and being in level 3. As will be proposed in the conclusions, this may be a future research work, carrying out a study with more values in the sample. 
TABLE 3: Summary of the tasks improved with the use of LAoCA by levels.

\begin{tabular}{lccc}
\hline & Easy task & Medium task & Difficult task \\
\hline Level 1 & ${ }^{* *}$ & ${ }^{* *}$ & $* *$ \\
Level 2 & ${ }^{* *}$ & ${ }^{* *}$ & - \\
Level 3 & ${ }^{* *}$ & - & - \\
Level 4 & - & - &
\end{tabular}

TABle 4: Summary of detection errors produced during experimentation.

\begin{tabular}{lccc}
\hline & Total number of movements & Total number of errors & \% of errors \\
\hline Easy task & 80 & 5 & 6.25 \\
Medium task & 80 & 7 & 8.75 \\
Difficult task & 128 & 12 & 9.375 \\
Total & 288 & 24 & 8.33 \\
\hline
\end{tabular}

TABLE 5: Summary of detection errors produced in experimentation excluding persons on level 4.

\begin{tabular}{lccc}
\hline & Total number of movements & Total number of errors & \% of errors \\
\hline Easy task & 60 & 1 & 1.67 \\
Medium task & 60 & 2 & 3.33 \\
Difficult task & 96 & 4 & 4.17 \\
Total & 216 & 7 & 3.24 \\
\hline
\end{tabular}

Finally, in Table 3, we show a summary of the tasks that underwent a statistically significant improvement by levels using our proposal.

5.2. Does It Improve Control over the Performed Tasks? In order to answer this research question, we analyzed two types of sources of information. The first one was a study of sensor failures about detecting the movements of tagged objects on the worktable, and the second one was a survey on the opinion of trainers in the execution of the experimentation. Our approach presented in this research work, in very few occasions, had problems to detect the movements of the tagged objects in the worktable. Most of the cases were due to the fact that objects were not placed exactly in the detection zones, and this was due to two main reasons:

(1) The objects are labeled with a small RFID tag in the center of the object, and the detection areas of the worktable have a very specific detection field.

(2) The participants did not place the objects exactly on the detection area.

Since this is a controlled experiment, we were able to measure the detection errors that were produced, below we present a study of these errors. Table 4 shows a summary of the detection errors in all the experimentation presented by tasks; as we can see, the total percentage of detection errors is 8.33 , which is above $5 \%$ of errors that would be acceptable.

During the experimentation, we detected that people with a higher level of disability produced more errors of detection of the objects; since, as we have said before, the system detects the movement if the object is placed in the detection zone correctly, which was not always the case with these people. That is why we excluded people on level 4 of the study, and the results are shown in Table 5. The number of detection errors was drastically reduced for the group consisting of people on levels 1,2, and 3. The total percentage of errors was 3.24, which is quite acceptable.

To complete this study, we also received the opinion of the trainers who helped us to carry out the experimentation of this research. Although we only had the information provided by three trainers, his opinion has been very valuable. In their opinion, the greatest contribution of this system is the possibility of tracing object movements on the worktable and the ability to record it for future analyze; or even that information can be served over the Internet in real time. Therefore, and in the opinion of trainers, LAoCA enables control and tracking capabilities; but as demonstrated by experimentation, it must be improved. These future improvements, further LAoCA versions, and experiments will be discussed in next section.

5.3. Is LAoCA Accepted by the Final Users? In this section, we present a study of the satisfaction perceived by the final users. In this research, there are two types of end users: participants and trainers. From participants, we wanted to find out two issues mainly: satisfaction and stress using LAoCA. The satisfaction was asked directly by the trainers to each of the participants after they finished the tasks, and the 
stress level was analyzed by the trainers for each of the participants and agreed among them.

As Figure 14 shows the level of satisfaction is quite high, with an average of 4.125 . This is because the participants took the experimentation as a game, since the worktable is quite attractive for the participants with four areas of detection of very striking colors, as well as the objects of detection that had different shapes and sizes. The participants left very enthusiastic and satisfied of the experimentation. However, during the experimentation, the participants suffered high degrees of stress as we can see in Figure 14; the mean of the stress level was 3.125. The participants did the experimentation one by one, in a room with three trainers observing the actions performed with the worktable and with several computers; as will be seen in Conclusions, it is recommended to perform this type of experimentation in a more environment-friendly manner for the participants. In addition, most of the participants presented their displeasure with the cyber-glove, which increased their level of stress and made them lose the attention of the tasks to be performed.

Also in this section, we present a study of the subjective perception of our approach from the point of view of trainers who participated in the experimentation. Because there were only three trainers who participated in the experimentation, we have sought the collaboration of seven other people to conduct a relevant survey about their opinion of our approach. These seven new people surveyed are specialists in education issues and described the qualities of LAoCA as they did a small test. Figure 15 shows a brief summary of the subjective evaluation of the ten specialists on three specific issues: utility, relevance, and satisfaction of our approach.

\section{Conclusions and Future Work}

In this research work, we present the second version of an approach for improving learning tasks for mentally handicapped people based on cyber-physical systems. The experimentation was carried out with the help and collaboration of the PRODIS Foundation, one of the most important foundations for mentally handicapped people in Spain. This research paper is an extended version of our previous work [6] presented in 10th International Conference on Ubiquitous Computing and Ambient Intelligence (UCAmI 2016). Our approach is called LAoCA, and it is composed mainly of three elements: a worktable, a cyberglobe, and a software for modeling and executing workflows. LAoCA was built following main techniques on cyberphysical systems, ambient intelligence, and Internet of things. In order to validate our proposal, we designed an experimentation involving sixteen people with mental disabilities and their trainers. This experimentation tries to answer three research questions presented in Introduction. The aim of the experiment, proposed in this research work, was to test whether the experiment participants improved their learning of tasks using our proposal. For this, they had to repeat a sequence of movements with the tagged objects on the worktable. We will use research questions in order to guide this section, and at the end, we will present a set of lessons learned after experimentation.

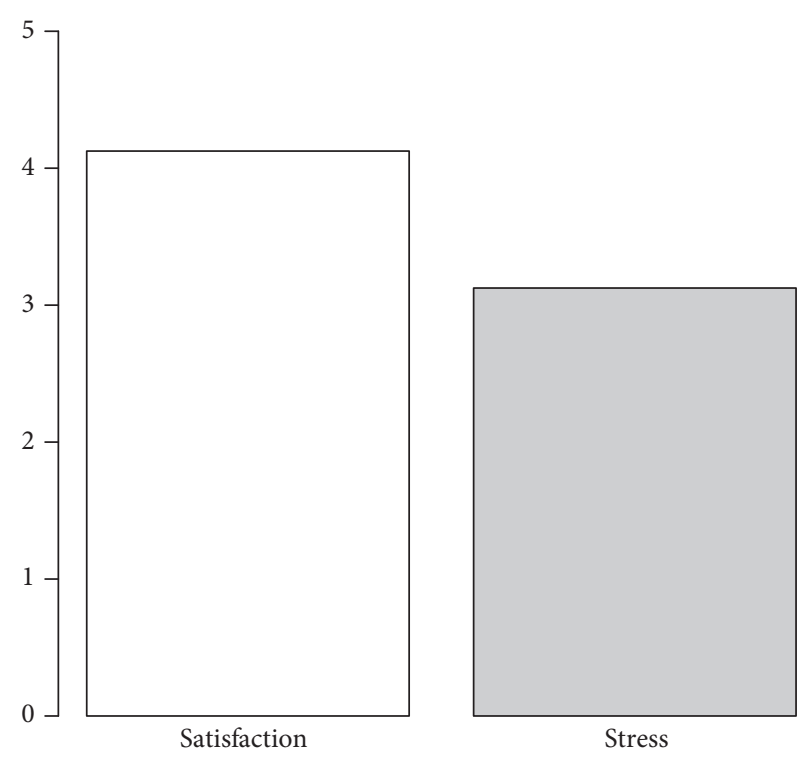

FIgURE 14: Evaluation of satisfaction and stress of the participants.

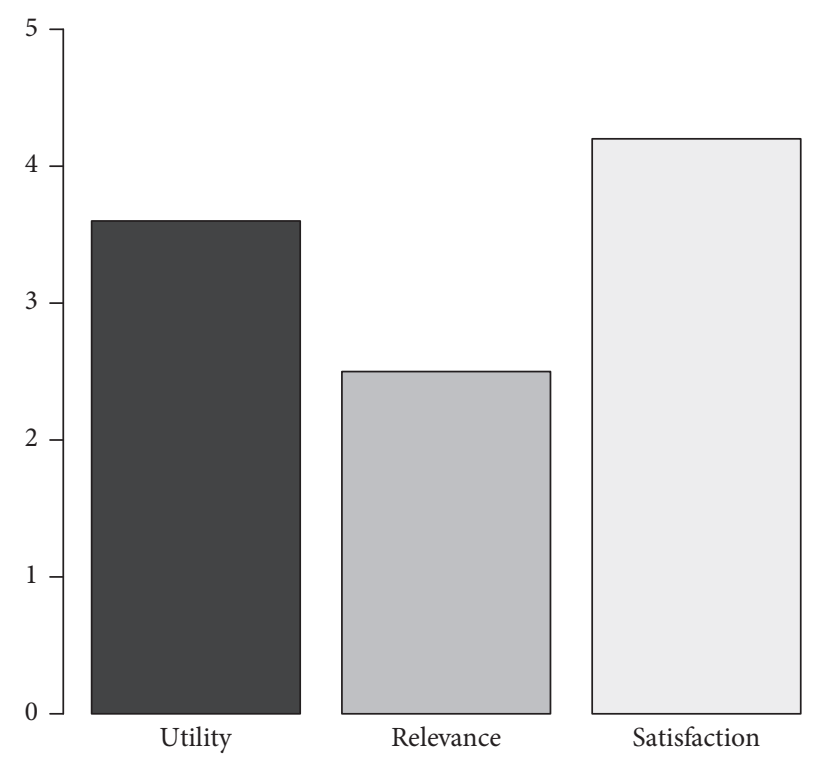

FIGURE 15: Evaluation of utility, relevance, and satisfaction of the trainers.

6.1. Can LAoCA Facilitate Learning for Mentally Handicapped People? Studying all data from the experimentation indicates that there is no significant improvement in participants learning as all levels of disability are studied in the same data set. Analyzing the data in more depth, it can be observed that not all the people are able to improve their learning as it depends on the level of disability. Excluding people on levels 3 and 4 of the experiment, we can see that there is an improvement in learning for people on levels 1 and 2 (i.e., people with less disability). For people on levels 1 and 2, using a classical training, participants had trouble completing the tasks; however, using LAoCA, they performed less errors. For this reason, we can assume that an AmI system based on CPS techniques can be useful for 
learning tasks for mentally handicapped people, but always bearing in mind that it is not easily applicable for people with a high level of disability. We also found a significant improvement for people on level 2 with easy task, but the statistical test had very little power because the sample is very small. This could be a point for further investigation. Therefore, the first conclusion we can draw is that not all people with mental disabilities are apt to use such devices; it is very important to know how to choose the set of people to work with, analyzing their level of disability and their ability to adapt to these devices. A possible future work would be to analyze how learning improves with people without mental disabilities, children, the elderly, etc.

6.2. Does It Improve Control over the Performed Tasks? To answer this research question, two sources of information have been studied: the first was a study about the detection failure from the sensors of LAoCA and the second was a survey of trainers about the benefits of it. Our approach sometimes does not detect the movements of tagged objects because the object is not placed in the center of the detection zone. We measured the number of detection failures and obtained that $8.33 \%$ of the movements are not detected correctly by LAoCA, which is not an acceptable value. But we realized that people on level 4 produced many detection errors, so we removed them from the study. In this way, the percentage of detection errors was reduced to 3.24. The conclusion that can be drawn reinforces that of the previous research question: not all people with disabilities can work with this type of device. The conclusion of the trainers is that the worktable is a good solution for control tasks and traceability and allows to control ambient assisted living tasks remotely, analyze the actions carried out a posteriori, and could even be developed an intelligent system that recognizes patterns in order to anticipate to critical situations.

6.3. Is LAoCA Accepted by the Final Users? In this section, we studied the level of satisfaction of the two groups of people that we consider the end users: the participants and the trainers. From the point of view of the participants, the level of satisfaction has been very high since the work done at the table was interpreted as a game. However, the participants according to their coaches suffered a lot of stress coming from a lack of familiarity with the participants. For future experiments, we propose two solutions: the first one is to create a familiar atmosphere with participants and the second consists to introduce new sensors to measure stress, blood pressure, sweat, etc. With these new sensors, we could know if the participant is suffering stress and we could stop the experimentation. From the point of view of the trainers, the utility, relevance, and satisfaction were studied, resulting in very high values for all parameters.

6.4. Lessons Learned after the Experiment Execution. After the execution of the experimentation with mentally handicapped people, we have drawn several conclusions that we would like to emphasize in this section. Regarding the cyberglove, it was an element of stress and loss of attention in most participants. We realized that something that is so attached to the body is not accepted by participants, generating doubts and rejection. That is why we recommend the use of elements that are as seamless as possible. As future work, we propose the development of a bracelet placed on the wrist able to detect objects caught with that hand. With respect to the worktable, the participants were distracted by the way it was built. For example, we used rivets to join the different pieces that make up the box. Those rivets were a source of distraction for the participants; most of them touched the rivets one by one until they discovered that they were not buttons.

In short and as a general conclusion, the system may be useful for people with a medium or low disability and for people who are not affected by other people or the environment. If the disability is more acute, it would require the presence of one or more evaluators. However, for people with a high disability or high concentration problems, this type of systems will not be useful since the system itself would be a source of distraction.

It is proposed as possible future work for the validation of each of the characteristics of the system proposed in this research work.

\section{Conflicts of Interest}

The authors declare that there are no conflicts of interest regarding the publication of this paper.

\section{Acknowledgments}

This research has received funding from the Ministry of Economy and Competitiveness through the INPAINK (RTC-2016-4881-7) and SEMOLA (TEC2015-68284-R) projects, this work was also partially supported by the Autonomous Region of Madrid through program MOSIAGIL-CM (grant P2013/ICE-3019, co-funded by EU Structural Funds FSE and FEDER).

\section{References}

[1] E. A. Lee, "Cyber-physical systems-are computing foundations adequate?," in Proceedings of the Position Paper for NSF Workshop on Cyber-Physical Systems: Research Motivation, Techniques and Roadmap, vol. 2, Austin, TX, USA, 2006.

[2] B. Bordel, R. Alcarria, T. Robles, and D. Martín, "Cyberphysical systems: extending pervasive sensing from control theory to the internet of things," Pervasive and Mobile Computing, vol. 40, pp. 156-184, 2017.

[3] B. Bordel Sánchez, R. Alcarria, D. Martín, and T. Robles, "TF4SM: a framework for developing traceability solutions in small manufacturing companies," Sensors, vol. 15, no. 11, pp. 29478-29510, 2015.

[4] G. Schirner, D. Erdogmus, K. Chowdhury, and T. Padir, "The future of human-in-the-loop cyber-physical systems," Computer, vol. 46, no. 1, pp. 36-45, 2013.

[5] D. Martín, R. Alcarria, Á. Sánchez-Picot, and T. Robles, “An ambient intelligence framework for end-user service 
provisioning in a hospital pharmacy: a case study, Journal of Medical Systems, vol. 39, no. 10, p. 116, 2015.

[6] D. Martín, B. Bordel, R. Alcarria, Á. Sánchez-Picot, D. S. de Rivera, and T. Robles, "Improving learning tasks for mentally handicapped people using AmI environments based on cyberphysical systems," in Proceedings of the 10th International Conference on Ubiquitous Computing and Ambient Intelligence (UCAmI 2016), Part I 10, pp. 166-177, Springer, San Bartolomé de Tirajana, Gran Canaria, Spain, November 2016.

[7] D. J. Cook and S. K. Das, "How smart are our environments? An updated look at the state of the art," Pervasive and Mobile Computing, vol. 3, no. 2, pp. 53-73, 2007.

[8] National Institute of Standards and Technology, "CPS public working group presentation,” 2015, http://www.nist. gov/el/upload/CPS-PWG-Kickoff-Webinar-PresentationFINAL.PDF.

[9] D. Sanchez De Rivera, R. Alcarria, D. Martin de Andres, B. Bordel, and T. Robles, "An autonomous information device with e-paper display for personal environments," in Proceedings of the 2016 IEEE International Conference on Consumer Electronics (ICCE), pp. 139-140, IEEE, Las Vegas, NV, USA, 2016.

[10] D. Sánchez-de Rivera, D. Martín, R. Alcarria, B. Bordel, and T. Robles, "Towards a wireless and low-power infrastructure for representing information based on e-paper displays," Sustainability, vol. 9, no. 1, p. 76, 2017.

[11] International Electrotechnical Commission, EnterpriseControl System Integration-Part 1: Models and Terminology, ANSI/ISA-95.00.01-2010 (IEC 62264-1 Mod), American National Standards Institute and Instrumentation, Systems, and Automation Society, Research Triangle Park, NC, USA, 2010.

[12] ACR Advisory Group, "Automation and software expenditures for discrete industries," 2015, http://www.arcweb. com/market-studies/pages/automation-expenditures-fordiscrete-industries.aspx.

[13] K. Thramboulidis and A. Zoupas, "Real-time java in control and automation: a model driven development approach," in Proceedings of the 10th IEEE Conference on Emerging Technologies and Factory Automation (ETFA 2005), vol. 1, pp. 846, IEEE, Catania, Italy, 2005.

[14] M. Jimenez, F. Rosique, P. Sanchez, B. Alvarez, and A. Iborra, "Habitation: a domain-specific language for home automation," IEEE Software, vol. 26, no. 4, pp. 30-38, 2009.

[15] OPC Foundation, OPC 2.1 Standard, 1996, https: //opcfoundation.org/.

[16] M. Philipose, K. P. Fishkin, M. Perkowitz et al., "Inferring activities from interactions with objects," IEEE Pervasive Computing, vol. 3, no. 4, pp. 50-57, 2004.

[17] C. Lee, M. Kim, J. Park, J. Oh, and K. Eom, "Design and implementation of the wireless RFID glove for life applications," International Journal of Grid and Distributed Computing, vol. 3, no. 3, pp. 41-52, 2010.

[18] K. P. Fishkin, M. Philipose, and A. Rea, "Hands-on RFID: wireless wearables for detecting use of objects," in Proceedings of the Ninth IEEE International Symposium on Wearable Computers (ISWC'05), pp. 38-41, IEEE, Washington, DC, USA, 2005.

[19] J. Lee, B. Bagheri, and H. A. Kao, "A cyber-physical systems architecture for industry 4.0-based manufacturing systems," Manufacturing Letters, vol. 3, pp. 18-23, 2015.

[20] G. Schuh, T. Gartzen, T. Rodenhauser, and A. Marks, "Promoting work-based learning through INDUSTRY 4.0," Procedia CIRP, vol. 32, pp. 82-87, 2015.
[21] S. Katz, A. B. Ford, R. W. Moskowitz, B. A. Jackson, and M. W. Jaffe, "Studies of illness in the aged: the index of ADL: a standardized measure of biological and psychosocial function," JAMA, vol. 185, no. 12, pp. 914-919, 1963.

[22] A. E. Majoros, B. C. Fredgren, P. R. Davies, and R. D. Kalinowski, "Data interface process with RFID data reader glove," US Patent 8482412, 2013.

[23] L. Atallah, B. Lo, R. King, and G. Z. Yang, "Sensor positioning for activity recognition using wearable accelerometers," IEEE Transactions on Biomedical Circuits and Systems, vol. 5, no. 4, pp. 320-329, 2011.

[24] J. Hallam and V. Whiteley, "Interactive therapy gloves: reconnecting partners after a stroke," in Proceedings of the 2011 Annual Conference Extended Abstracts on Human Factors in Computing Systems (CHI EA'11), pp. 989-994, ACM, Vancouver, BC, Canada, 2011.

[25] M. Konkel, V. Leung, B. Ullmer, and C. Hu, "Tagaboo: a collaborative children's game based upon wearable RFID technology," Personal and Ubiquitous Computing, vol. 8, no. 5, pp. 382-384, 2004.

[26] L. Escobedo, C. Ibarra, J. Hernandez, M. Alvelais, and M. Tentori, "Smart objects to support the discrimination training of children with autism," Personal and Ubiquitous Computing, vol. 18, no. 6, pp. 1485-1497, 2014.

[27] Lemelson MIT Program, "SignAloud: gloves that transliterate sign language into text and speech," 2016, https://www. youtube.com/watch?v=101sdzJHCCM.

[28] P. S. López, J. Llinás, J. Amiel, C. Vidal, and J. Vilalta, "CAMDEX: a new psychogeriatric interview," Actas LusoEspanolas de Neurologia, Psiquiatria y Ciencias Afines, vol. 18, no. 5, pp. 290-295, 1989. 


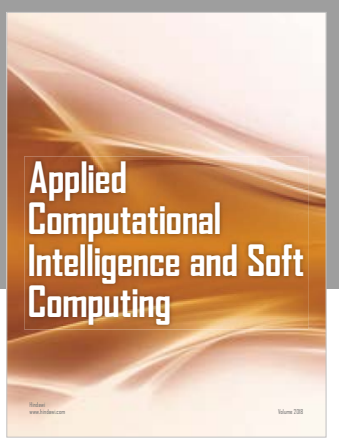

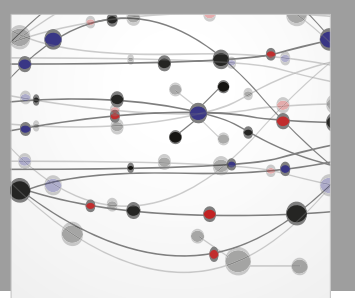

The Scientific World Journal
Submit your manuscripts at

Computing
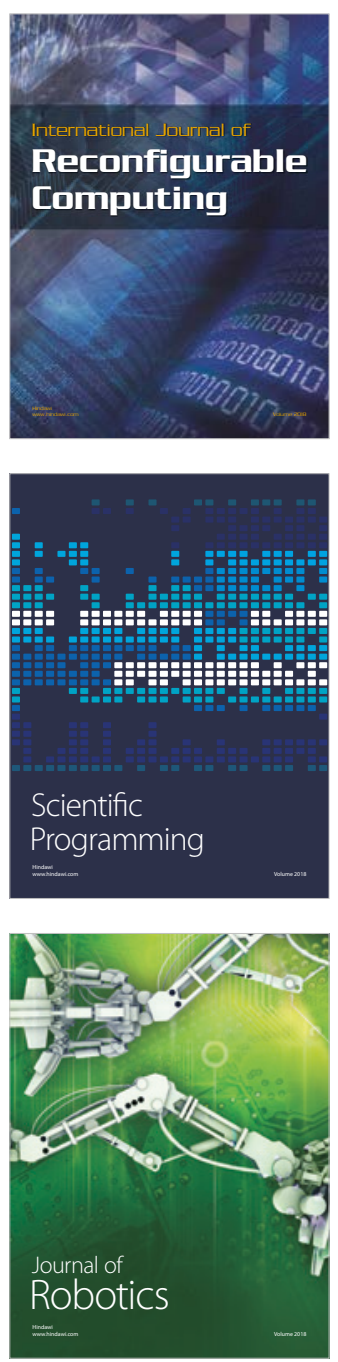

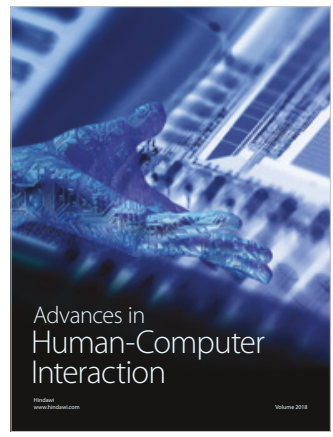

Human-Compute

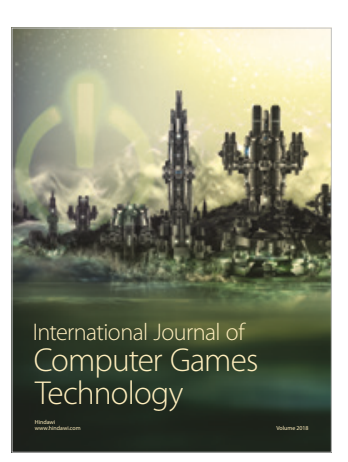

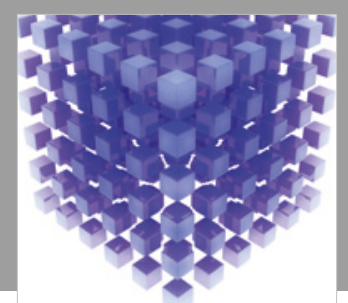

Mathematical Problems in Engineering

\section{Engincering}
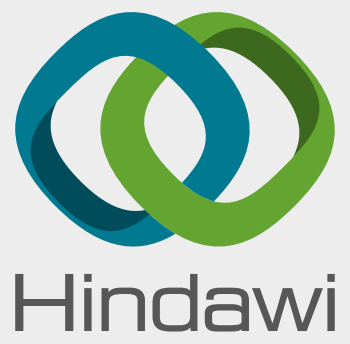

www.hindawi.com
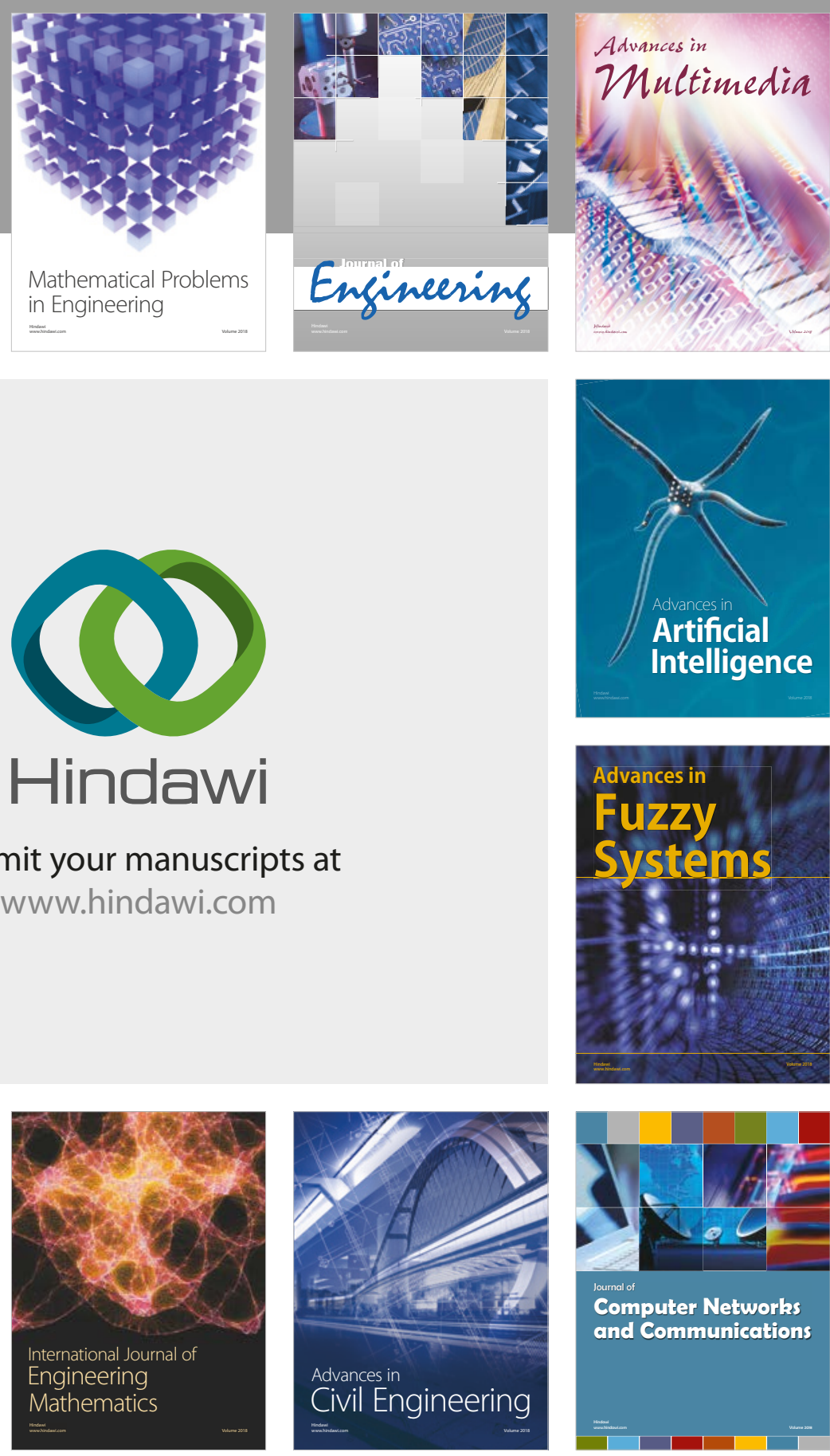

Computer Networks and Communications

Multimedia
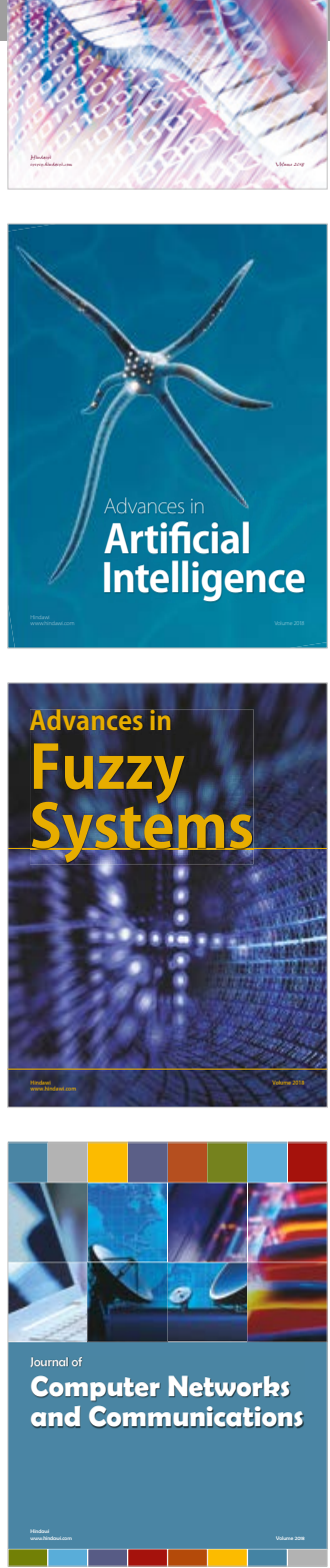

Advances in

Modelling \&

Simulation

in Engineering

interaction

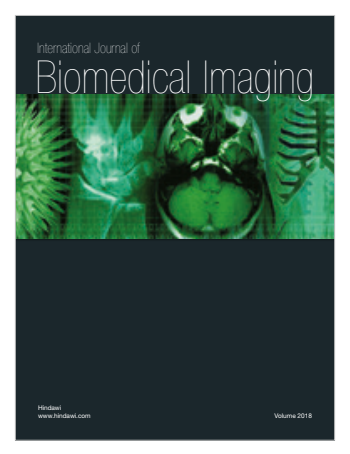

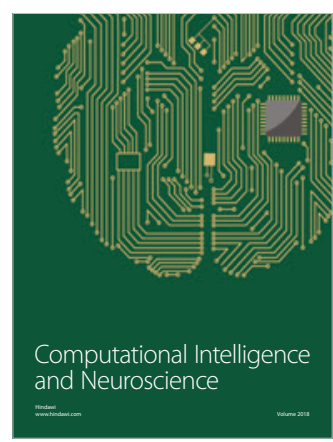

